\title{
Numerical analysis of the impact of the ion threshold, ion stiffness and temperature pedestal on global confinement and fusion performance in JET and in ITER plasmas
}

\author{
Baiocchi, B.; Mantica, P.; Tala, T.; Corrigan, G.; Joffrin, E.; Kirov, K.; Naulin, Volker
}

Published in:

Plasma Physics and Controlled Fusion

Link to article, DOI:

$10.1088 / 0741-3335 / 54 / 8 / 085020$

Publication date:

2012

Document Version

Publisher's PDF, also known as Version of record

Link back to DTU Orbit

Citation (APA):

Baiocchi, B., Mantica, P., Tala, T., Corrigan, G., Joffrin, E., Kirov, K., \& Naulin, V. (2012). Numerical analysis of the impact of the ion threshold, ion stiffness and temperature pedestal on global confinement and fusion performance in JET and in ITER plasmas. Plasma Physics and Controlled Fusion, 54, 085020. https://doi.org/10.1088/0741-3335/54/8/085020

\section{General rights}

Copyright and moral rights for the publications made accessible in the public portal are retained by the authors and/or other copyright owners and it is a condition of accessing publications that users recognise and abide by the legal requirements associated with these rights.

- Users may download and print one copy of any publication from the public portal for the purpose of private study or research.

- You may not further distribute the material or use it for any profit-making activity or commercial gain

- You may freely distribute the URL identifying the publication in the public portal 
Numerical analysis of the impact of the ion threshold, ion stiffness and temperature pedestal on global confinement and fusion performance in JET and in ITER plasmas

This article has been downloaded from IOPscience. Please scroll down to see the full text article.

2012 Plasma Phys. Control. Fusion 54085020

(http://iopscience.iop.org/0741-3335/54/8/085020)

View the table of contents for this issue, or go to the journal homepage for more

Download details:

IP Address: 192.38.67.112

The article was downloaded on 19/07/2012 at 13:49

Please note that terms and conditions apply. 


\title{
Numerical analysis of the impact of the ion threshold, ion stiffness and temperature pedestal on global confinement and fusion performance in JET and in ITER plasmas
}

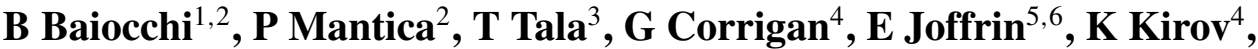 \\ $V$ Naulin $^{7}$ and JET-EFDA contributors ${ }^{8}$ \\ JET-EFDA, Culham Science Centre, OX14 3DB, Abingdon, UK \\ ${ }^{1}$ Università degli Studi di Milano, Milano, Italy \\ ${ }^{2}$ Istituto di Fisica del Plasma 'P Caldirola', Associazione Euratom-ENEA-CNR, Milano, Italy \\ ${ }^{3}$ Association Euratom-Tekes, VTT, PO Box 1000, FIN-02044 VTT, Finland \\ ${ }^{4}$ Euratom/CCFE Association, Culham Science Centre, Abingdon, OX14 3DB, UK \\ ${ }^{5}$ CEA, IRFM, F-13108 Saint Paul Lez Durance, France \\ ${ }^{6}$ EFDA-CSU, Culham Science Centre, OX14 3DB, Abingdon, UK \\ ${ }^{7}$ Association Euratom-Ris $\varnothing$ DTU, PLF-128 Ris $\varnothing$, DK-4000 Roskilde, Denmark
}

Received 14 July 2011, in final form 5 February 2012

Published 16 July 2012

Online at stacks.iop.org/PPCF/54/085020

\begin{abstract}
Understanding the impact of micro-instabilities on the global plasma performance is essential in order to make realistic predictions for relevant tokamak scenarios. The semi-empirical transport model CGM is a useful tool to this scope because it depends explicitly on the threshold and the stiffness level, two key parameters of turbulent transport as driven by the ITG/TEM instabilities. The CGM then makes it possible to vary separately the transport variables and to see the quantitative effect of their changes on the global plasma performance. This paper focuses on the impact that four parameters (ion temperature gradient threshold, ion temperature gradient stiffness, height of the temperature pedestal and input power) have individually on the global confinement. Parameters for JET hybrid plasmas and prospective ITER plasmas are used. For JET plasmas changing the ion temperature gradient stiffness from typical low values (characterized by $\left.\chi_{\mathrm{s}}=0.1\right)$ to high values $\left(\chi_{\mathrm{s}}=2\right)$ leads to variations in the $H$ factor up to $30 \%$. Varying the ion temperature gradient threshold within the interval of the realistic values 3-7 causes changes in $H_{98}$ between $20 \%$ and $30 \%$. The effect of the temperature pedestal height is very considerable (over $50 \%$ of $H_{98}$ variation changing the temperature pedestal height from 1 to $3 \mathrm{keV}$ ), in agreement with the previous investigations. $H_{98}$ is found to slightly decrease with increasing power (from 20 to $60 \mathrm{MW}$ of injected NBI power) for high stiffness and to remain constant in the case of low stiffness. For ITER plasmas the variation of the $H$ factor has qualitatively similar trends, but the variations with respect to changes in stiffness and threshold are smaller. However, very important changes are found for the values predicted by the fusion power in these plasmas.
\end{abstract}

(Some figures may appear in colour only in the online journal)

\section{Introduction}

The turbulent character of the heat transport in tokamak plasmas has been intensively investigated through experimental

\footnotetext{
${ }^{8}$ See the appendix of Romanelli F et al 2010 Proc. 23rd IAEA Fusion Energy Conf. 2010 (Daejeon, Korea).
}

studies and it seems to be ascribed to ITG/TEM instabilities [1-3]. Theoretically they are described to take place above a threshold value of the quantity $R / L_{T}$, where $R$ is the major radius, $L_{\mathrm{T}}=T /|\operatorname{grad} T|$ is the characteristic gradient length of the temperature $T$. Above this value the heat flux of the plasma grows strongly with increasing $R / L_{T}$, assuming values much higher than predicted by the neoclassical transport theory. As 
a consequence the $T$ profiles cannot be raised significantly above their threshold values: this property is called stiffness of $T$ profiles. In particular, we refer to an high level of stiffness when the heat flux growth implies a minimum variation of $R / L_{T}$, which keeps very close to the threshold value. In contrast, if the increase in the heat flux leads to a significant growth of $R / L_{T}$, this trend is identified with a low level of stiffness.

The above described behaviour has been verified experimentally for electrons [4]: electron threshold and electron stiffness factor were measured in perturbative experiments, and comparison with theory and parametric studies were widely carried out [5]. Recent experiments have led to the first comparison of theoretical predictions for threshold and stiffness also for ions. A correlation between edge and core $T_{\mathrm{i}}$ values $[6,7]$ and a substantial change in slope at the threshold of the $q_{\mathrm{i}}$ versus $R / L_{T_{\mathrm{i}}}$ curve, built using their radial excursion [8-10], have been experimentally observed. Through $T_{\mathrm{i}}$ modulation experiments the first measurements of the ion stiffness level have been obtained, and experimental studies performed at various radial positions have led to building the $q_{\mathrm{i}}$ versus $R / L_{T_{\mathrm{i}}}$ curve at a given radius keeping all the parameters unchanged, allowing the determination of the local threshold and stiffness level [11]. Studies of parametric dependences of ion threshold and stiffness have then been made [12,13]. It is important to link these ion transport studies about ITG threshold and stiffness, two important internal properties of turbulence driven transport, with the investigation of plasma scenarios, in order to understand if they can be useful knobs to improve the scenario performance. In this paper, we focus on the investigation of the effect that some parameters connected with the ion transport have individually on the plasma confinement. To this scope series of simulations have been made using the transport model critical gradient model (CGM) [14]. It is a semi-empirical tool which depends explicitly on threshold and stiffness. It has been implemented in JETTO, a $1.5 \mathrm{D}$ core transport code [15]. We describe the results obtained by numerical analysis work scanning the four parameters ion temperature gradient threshold, ion temperature gradient stiffness, height of ion and electron temperature pedestal and input power, in order to give a quantitative evaluation of the impact that each of them has separately on the global confinement. Particular attention has been given to the role of the ion stiffness level. While ion threshold and temperature pedestal height are known to have a significant impact on global plasma performance and are already taken into account for confinement optimization studies, the stiffness level is not much considered so far. JET hybrid plasmas have been used as the basis of the simulations and then the same numerical analysis has been applied to standard parameter ITER H-mode plasmas.

This paper is organized as follows: the CGM model is introduced and described in section 2. Then simulations and numerical analysis results are presented in section 3 for hybrid JET plasmas and in section 4 for ITER plasmas. The paper ends with the summary and conclusions in section 5 .

\section{Critical gradient model}

The majority of existing first-principles transport models feature the existence of a threshold above which the transport switches from residual to highly turbulent. However, each of them shows a different sensitivity to the threshold. In order to quantify the above-threshold plasma behaviour, the semi-empirical transport model CGM was proposed $[14,16]$. It covers the basic properties of turbulent transport though not all the physics known from first-principles turbulence simulations. The CGM parametrizes turbulent transport into a handful of variables. In particular, it depends explicitly on threshold and stiffness. This parametrization makes possible easy comparison with experiments, and also provides easy access to the scaling laws of global confinement.

It was initially developed for electron heat transport and it has recently been used also for ions. The CGM is based on the existence of an instability threshold in the inverse temperature gradient length and of finite background transport below the threshold. In addition, it is characterized by an electrostatic gyro-Bohm scaling law. This latter assumption relies on theory and scaling studies and is assumed valid in the limit of a small normalized gyro-radius [17-19].

In the CGM model the ion heat flux is written as

$$
\begin{aligned}
q_{\mathrm{i}}^{\mathrm{gB}}= & n_{\mathrm{i}} q^{1.5} \chi_{\mathrm{si}} \frac{T_{\mathrm{i}}^{2} \rho_{\mathrm{i}}}{e B R^{2}} f\left[\frac{R}{L_{T_{\mathrm{i}}}}-\left(\frac{R}{L_{T_{\mathrm{i}}}}\right)_{\mathrm{cr}}\right] \\
& \times H\left[\frac{R}{L_{T_{\mathrm{i}}}}-\left(\frac{R}{L_{T_{\mathrm{i}}}}\right)_{\mathrm{cr}}\right] \frac{R}{L_{T_{\mathrm{i}}}}+q_{\mathrm{i}}^{\mathrm{res}} .
\end{aligned}
$$

Here $q_{\mathrm{i}}^{\text {res }}$ is the ion residual flux, including the neoclassical flux, $\chi_{\mathrm{si}}$ is the ion stiffness factor; it quantifies the stiffness level and characterizes how strongly $T_{\mathrm{i}}$ profiles are tied to the threshold. The dependence of the heat flux on $q$, the safety factor, allows recovery of the experimentally observed dependence of confinement on plasma current and radial increase in transport from core to edge. $n_{\mathrm{i}}$ is the ion density, $T_{\mathrm{i}}$ is the ion temperature, $e$ is the electron charge, $B$ is the magnetic field, $R$ is the major radius. $\rho_{\mathrm{i}}=\left(m_{\mathrm{i}} T_{\mathrm{i}}\right)^{1 / 2} / e B$ is the ion Larmor radius, where $m_{\mathrm{i}}$ is the ion mass. $H$ is the Heaviside step function, which gives the threshold effect above a critical value of $R / L_{T_{\mathrm{i}}},\left(R / L_{T_{\mathrm{i}}}\right)_{\mathrm{cr}}$. The theory foresees $q_{\mathrm{i}}$ to be linear with $R / L_{T_{\mathrm{i}}}$ [20], but it is valid only far from the threshold. In the experiments, not too far from the threshold, it is not possible to identify if the real dependence is linear or quadratic because of experimental uncertainties. Hence we remain coherent with the previous works on electron $[5,21]$ and ion $[11,21]$ stiffness, following the semi-empirical model described in [16], where $f\left(R / L_{T_{\mathrm{i}}}\right)$ is taken linear so that $q_{\mathrm{i}}$ is quadratic in $R / L_{T_{\mathrm{i}}}$.

\section{Ion stiffness, ion threshold, pedestal and power scans in JET hybrid plasmas}

\subsection{Simulation set-up}

Several simulations have been performed in order to scan four significant parameters: the ion temperature gradient threshold, the ion temperature gradient stiffness, the height of temperature pedestal and the input power. For each scan only one parameter 
has been varied while we have kept all the other quantities unchanged. In this context, each of the parameters varied is to be considered independent of all the others and the choice of the values for simulations is within realistic ranges and such that allows exploring satisfactorily the dependences under study. In order to follow this procedure the CGM has been used, after implementing it into the transport code JETTO ( [15], part of the JET JAMS suite of integrated codes). Geometrical and physical parameters have been taken from the hybrid JET shot number 77043 as the basis of the simulations. The hybrid scenario is an operation mode with reduced current and increased confinement, in which a substantial part of the plasma current is driven non-inductively. It is thought of and realized as an intermediate regime between standard $\mathrm{H}$-mode and true steady-state modes of operation [22]. Recent studies $[23,24]$ show that in hybrid discharges a relevant improvement of the confinement takes place. The core improvement has been ascribed to the reduction in the ion stiffness $[12,13]$.

It is reasonable to expect that the hybrid scenario is a regime in which the effect of the variation of the ion stiffness is more significant than in non-hybrid discharges because, following the interpretation of $[12,13]$, hybrids are characterized by a broader region in which improved ion temperature profiles are observed. The impact of the ion stiffness variation is expected significant also with respect to the effects on the confinement variation due to changes in the ion threshold and the height of temperature pedestal. The JET hybrid shot number 77043 is characterized by low density and low triangularity, with $B_{\mathrm{t}}=1.9 \mathrm{~T}, I_{\mathrm{p}}=1.7 \mathrm{MA}$, $Z_{\text {eff }}=2.3$. Beyond the above indicated parameters, the input data taken from the JET database include the equilibrium configuration, the geometry, the density profile (with $n_{\mathrm{e} 0}=$ $5.1 \times 10^{19} \mathrm{~m}^{-3}$ ), the monotonic $q$ profile (with $q_{0}=1.28$ ) and in addition the initial and boundary conditions for electron and ion temperatures, quantities considered predictive in this numerical work, i.e. calculated solving ion and electron heat transport equations. The plasma current has been treated as an interpretative quantity, taking directly the value of the experimental discharge 77043. The current density profile evolution is instead predicted using the JETTO internal equilibrium solver, ESCO. For this, all current sources have been considered, including the NBI driven component, which is about $15 \%$ of the total plasma current. NBI power deposition profiles have been taken from PENCIL code calculations [25]. The neoclassical ion heat transport is calculated using NCLASS, a transport code inside JETTO.

For the scans with fixed temperature pedestal height and/or power we started keeping the parameters typical of a JET hybrid plasma: $1.5 \mathrm{keV}$ for the temperature pedestal and 25.5 MW of NBI power. Then we modified these parameters, reducing the height of the temperature pedestal to $1 \mathrm{keV}$ or the power to $17 \mathrm{MW}$.

In scans with fixed ion stiffness and/or ion threshold we have chosen their values following the results obtained by recent heating power modulation experiments $[11,26]$, which allow us to distinguish the effects of stiffness and threshold, providing a direct measurement of them. In particular, we have performed simulations with ion stiffness and ion threshold
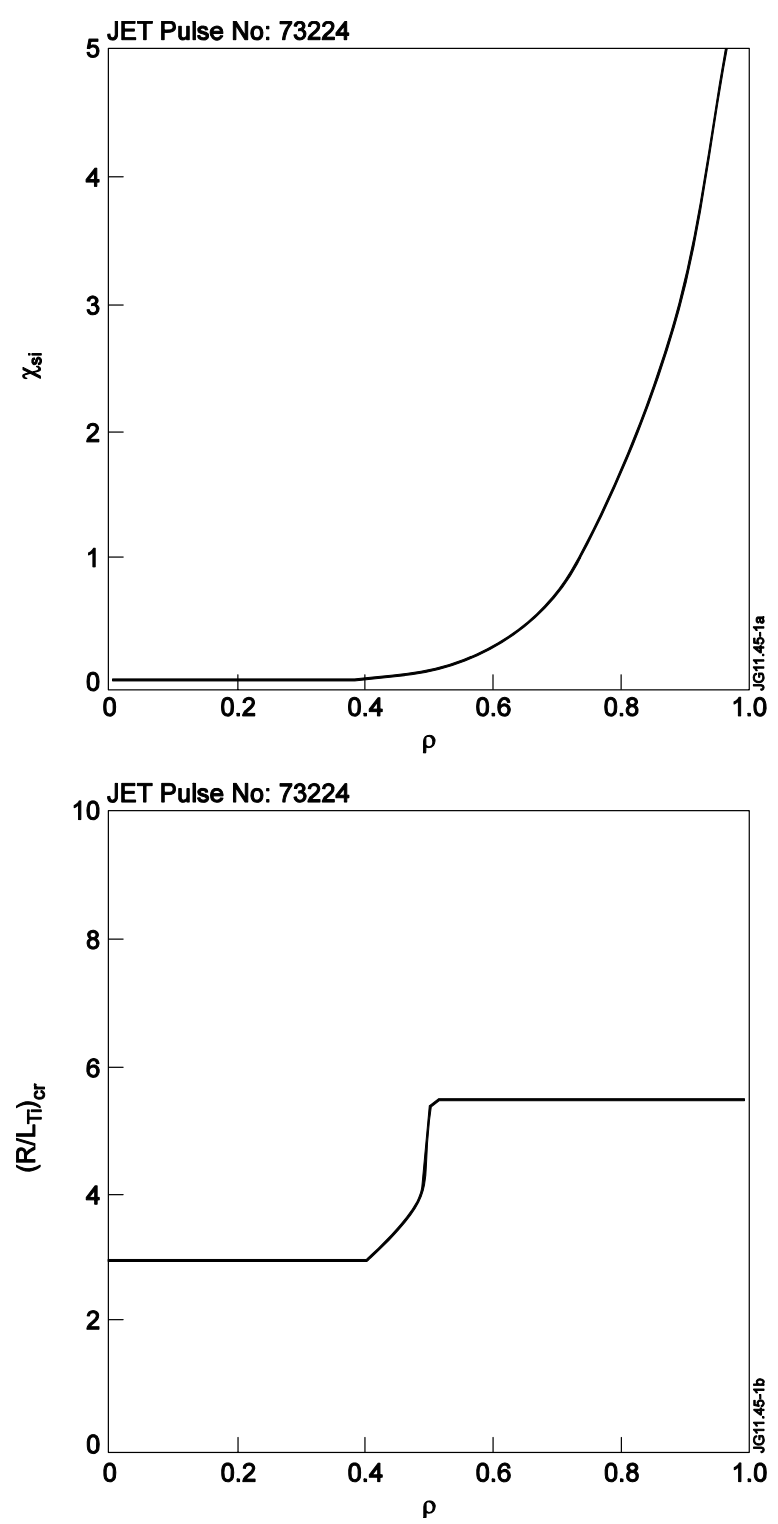

Figure 1. Ion stiffness $(a)$ and ion threshold $(b)$ profiles as obtained by modulation experiments.

constant over the radius, with ion threshold equal to 4 , and considering values of both high and low ion stiffness in order to take into account the broad range it covers in experiments. The modulation experiments analysis has led to determining ion stiffness and ion threshold radially varying profiles, which allow us to achieve a better match with the experimental data with respect to stiffness and threshold radially uniform values. According to these results we have repeated the scans using the radially dependent profiles of ion stiffness and ion threshold shown in figure 1 . In all the simulations presented in this paper, fixed values of threshold (equal to 5) and stiffness (equal to 1) have been taken for electrons following the recent results of perturbative experiments [21]. A list of the made scans is shown in table 1.

\subsection{Simulation results}

The simulation results can be described through the quantity $\mathrm{H}_{98}$ [27]. It is defined as the ratio of the thermal energy 
Table 1. Scans of ion stiffness, ion threshold, temperature pedestal and power for hybrid JET plasmas.

\begin{tabular}{|c|c|c|c|c|}
\hline & Ion stiffness & Ion threshold & Temperature pedestal & power \\
\hline Scan of ion stiffness & $\begin{array}{l}\text { from } 0.1 \text { to } 2 \\
\text { from } 0.1 \text { to } 2 \\
\text { from } 0.1 \text { to } 2 \\
\text { from } 0.1 \text { to } 2 \\
\text { from } 0.1 \text { to } 2 \\
\text { from } 0.1 \text { to } 2\end{array}$ & $\begin{array}{l}4 \\
4 \\
4 \\
\text { profile } \\
\text { profile } \\
\text { profile }\end{array}$ & $\begin{array}{l}1.5 \mathrm{keV} \\
1.5 \mathrm{keV} \\
\text { reduced, } 1 \mathrm{keV} \\
1.5 \mathrm{keV} \\
1.5 \mathrm{keV} \\
\text { reduced, } 1 \mathrm{keV}\end{array}$ & $\begin{array}{l}25.5 \mathrm{MW} \\
\text { reduced, } 17 \mathrm{MW} \\
25.5 \mathrm{MW} \\
25.5 \mathrm{MW} \\
\text { reduced, } 17 \mathrm{MW} \\
25.5 \mathrm{MW}\end{array}$ \\
\hline Scan of ion threshold & $\begin{array}{l}0.4 \\
0.4 \\
0.4 \\
2 \\
2 \\
2 \\
\text { profile } \\
\text { profile } \\
\text { profile }\end{array}$ & $\begin{array}{l}\text { from } 3 \text { to } 12 \\
\text { from } 3 \text { to } 12 \\
\text { from } 3 \text { to } 12 \\
\text { from } 3 \text { to } 12 \\
\text { from } 3 \text { to } 12 \\
\text { from } 3 \text { to } 12 \\
\text { from } 3 \text { to } 12 \\
\text { from } 3 \text { to } 12 \\
\text { from } 3 \text { to } 12\end{array}$ & $\begin{array}{l}1.5 \mathrm{keV} \\
1.5 \mathrm{keV} \\
\text { reduced, } 1 \mathrm{keV} \\
1.5 \mathrm{keV} \\
1.5 \mathrm{keV} \\
\text { reduced, } 1 \mathrm{keV} \\
1.5 \mathrm{keV} \\
1.5 \mathrm{keV} \\
\text { reduced, } 1 \mathrm{keV}\end{array}$ & $\begin{array}{l}25.5 \mathrm{MW} \\
\text { reduced, } 17 \mathrm{MW} \\
25.5 \mathrm{MW} \\
25.5 \mathrm{MW} \\
\text { reduced, } 17 \mathrm{MW} \\
25.5 \mathrm{MW} \\
25.5 \mathrm{MW} \\
\text { reduced, } 17 \mathrm{MW} \\
25.5 \mathrm{MW}\end{array}$ \\
\hline Scan of temperature pedestal & $\begin{array}{l}0.4 \\
0.4 \\
2 \\
2 \\
\text { profile } \\
\text { profile }\end{array}$ & $\begin{array}{l}4 \\
4 \\
4 \\
4 \\
\text { profile } \\
\text { profile }\end{array}$ & $\begin{array}{l}\text { from } 0.1 \text { to } 3 \mathrm{keV} \\
\text { from } 0.1 \text { to } 3 \mathrm{keV} \\
\text { from } 0.1 \text { to } 3 \mathrm{keV} \\
\text { from } 0.1 \text { to } 3 \mathrm{keV} \\
\text { from } 0.1 \text { to } 3 \mathrm{keV} \\
\text { from } 0.1 \text { to } 3 \mathrm{keV}\end{array}$ & $\begin{array}{l}25.5 \mathrm{MW} \\
\text { reduced, } 17 \mathrm{MW} \\
25.5 \mathrm{MW} \\
\text { reduced, } 17 \mathrm{MW} \\
25.5 \mathrm{MW} \\
\text { reduced, } 17 \mathrm{MW}\end{array}$ \\
\hline Scan of power & $\begin{array}{l}0.1 \\
0.1 \\
0.1 \\
2 \\
2 \\
2\end{array}$ & $\begin{array}{l}7 \\
4 \\
7 \\
7 \\
4 \\
7\end{array}$ & $\begin{array}{l}1.5 \mathrm{keV} \\
1.5 \mathrm{keV} \\
\text { reduced, } 1 \mathrm{keV} \\
1.5 \mathrm{keV} \\
1.5 \mathrm{keV} \\
\text { reduced, } 1 \mathrm{keV}\end{array}$ & $\begin{array}{l}\text { from } 17 \text { to } 60 \mathrm{MW} \\
\text { from } 17 \text { to } 60 \mathrm{MW} \\
\text { from } 17 \text { to } 60 \mathrm{MW} \\
\text { from } 17 \text { to } 60 \mathrm{MW} \\
\text { from } 17 \text { to } 60 \mathrm{MW} \\
\text { from } 17 \text { to } 60 \mathrm{MW}\end{array}$ \\
\hline
\end{tabular}

confinement time and the experimental scaling of energy confinement time of $\mathrm{H}$-mode plasmas. It depends on the physical quantities shown in formula (2).

$$
\begin{aligned}
H_{98} & =\frac{\tau_{E}}{\tau_{98}} \\
& =\frac{W_{\text {th }}}{0.0562 P^{0.31} I_{\mathrm{P}}^{0.93} B_{0}^{0.15} n^{0.41} R_{0}^{1.97} \kappa_{\mathrm{a}}^{0.78}\left(a_{0} / R_{0}\right)^{0.58} A^{0.19}} .
\end{aligned}
$$

Here $W_{\text {th }}$ is the total thermal energy, $P$ the total power (given by the sum of the ohmic power, the injected power and the $\alpha$ power), $I_{\mathrm{P}}$ the plasma current, $B_{0}$ the magnetic field, $n$ the density and $A$ the average ion mass. The $H$ factor depends also on geometrical parameters: the major radius $R_{0}$, the minor radius $a_{0}$, the elongation $\kappa_{\mathrm{a}}$ (defined as the ratio of the plasma volume and $2 \pi^{2} a_{0}^{2} R_{0}$ ). Normally $H_{98}$ is equal to one for $\mathrm{H}$-mode plasmas, while for hybrid plasmas it reaches higher values, $H_{98} \cong 1.4$ [28]. Our scan work aims not to obtain the typical hybrid plasma values of the $H$ factor (that depends for example on the shape of the electron temperature profile, which here is modeled only to be consistent with the resultant ion temperature profile), instead to quantify the changes in $H_{98}$ for varying ion heat transport.

We started with the scan of the ion temperature gradient stiffness. In figure 2 the $H$ factor is presented as a function of the ion stiffness for different values of the power or/and the ion and electron temperature pedestal height.

As expected, we can see that the $H$ factor, and then the confinement, decreases with increasing ion stiffness independently of pedestal and power values. It is verified

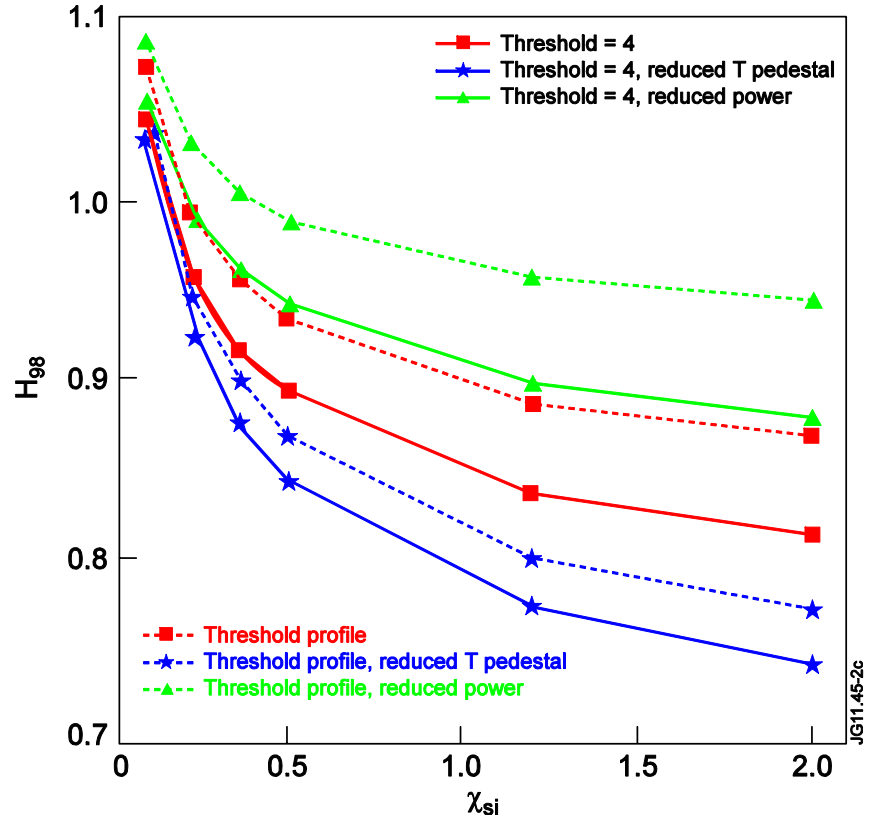

Figure 2. $H$ factor as a function of the ion stiffness level $\chi_{\mathrm{si}}$, for constant ion threshold (solid lines), for the threshold profile presented in figure 1 (dashed lines). The simulations with typical hybrid plasma temperature pedestal and power are shown with squares, those with reduced temperature pedestal with stars and the case with reduced power is represented with triangles.

for a fixed ion threshold constant in radius (solid lines) and with radial profile (dashed lines), though in this last case the $H$ factor is slightly less sensible to the stiffness changes. The variation of $H_{98}$ with ion stiffness is larger for the case 
of reduced temperature pedestal height and typical hybrid plasma power: changes from high stiffness to low stiffness increase the $H$ factor by $30 \%$ in the case of constant threshold equal to 4 . Reducing the pedestal height leads to lower temperature profiles, that however, for low ion stiffness, grow up to almost reach the profiles obtained for higher pedestal in the core region. For high stiffness the difference between the temperature profiles remains significant in both the edge and the core region. Hence in this last case we have an energy content very different for the high pedestal and the reduced pedestal simulations. $H_{98}$, which is directly proportional to the thermal energy, is then affected by this behaviour and decreases more in the case of reduced pedestal height. The variation of the $H$ factor is lower for a typical hybrid plasma temperature pedestal and power, and it is even lower in the case of reduced power. However, $H_{98}$ changes significantly also in this last case, by about $15 \%$. Reducing the injected power causes the decrease in the temperature profiles with respect to the case of typical hybrid power, and then of the thermal energy; however, the $H$ factor reaches higher values because it is also inversely proportional to the input power as formula (2) shows. The difference between the temperature profiles obtained changing the power is found lower for higher ion stiffness and leads to similar values of the thermal energy. Then $H_{98}$ varies less with the ion stiffness for the case of reduced power. The ion temperature profiles are shown in figure 3(b) and their inverse gradient lengths as a function of the stiffness level are shown in figure 3(a), for the case of typical hybrid plasma temperature pedestal height, equal to $1.5 \mathrm{keV}$, and power of $25.5 \mathrm{MW}$, with constant ion threshold equal to 4 . This behaviour is found similar in the simulations with other values of pedestal height, power and ion threshold.

Looking at figure $3(a)$, we can see that all the values assumed by $R / L_{T_{\mathrm{i}}}$ exceed the threshold $\left(\left(R / L_{T_{\mathrm{i}}}\right)_{\mathrm{cr}}=4\right)$ ). It means that the transport has always a turbulent component. If we look at $T_{\mathrm{i}}$ profiles shown in figure 3(b) for different values of the ion stiffness, we can see that the $T_{\mathrm{i}}$ value in the plasma centre varies significantly with increasing stiffness, from 7.6 to $5 \mathrm{keV}$.

Then we proceeded with the scan of the ion temperature gradient threshold, fixing all the other parameters. In figure 4 the results are shown.

We can see that the $H$ factor grows with increasing ion threshold, as expected. This is true for all the values chosen for the ion stiffness (constant or dependent on the radial coordinate), and for every considered temperature pedestal height and power. Increasing threshold makes a significant change in $\mathrm{H}_{98}$, both at typical hybrid parameters and reduced pedestal height and power, though its growth takes places differently for high threshold values. In particular, for the cases with hybrid plasma temperature pedestal height the increase in $H$ factor seems to saturate, especially for the scan with reduced power. We note in addition that, from linear gyrokinetic scans [29] and considering the threshold up-shift effect due to rotation through the Waltz rule [30], in the usual range of parameters of the H-mode or hybrid plasmas the ion threshold is typically below 7 . In this range the variation of $H_{98}$ is up to $30 \%$ with high stiffness (dashed lines), and about $20 \%$ in the other cases.
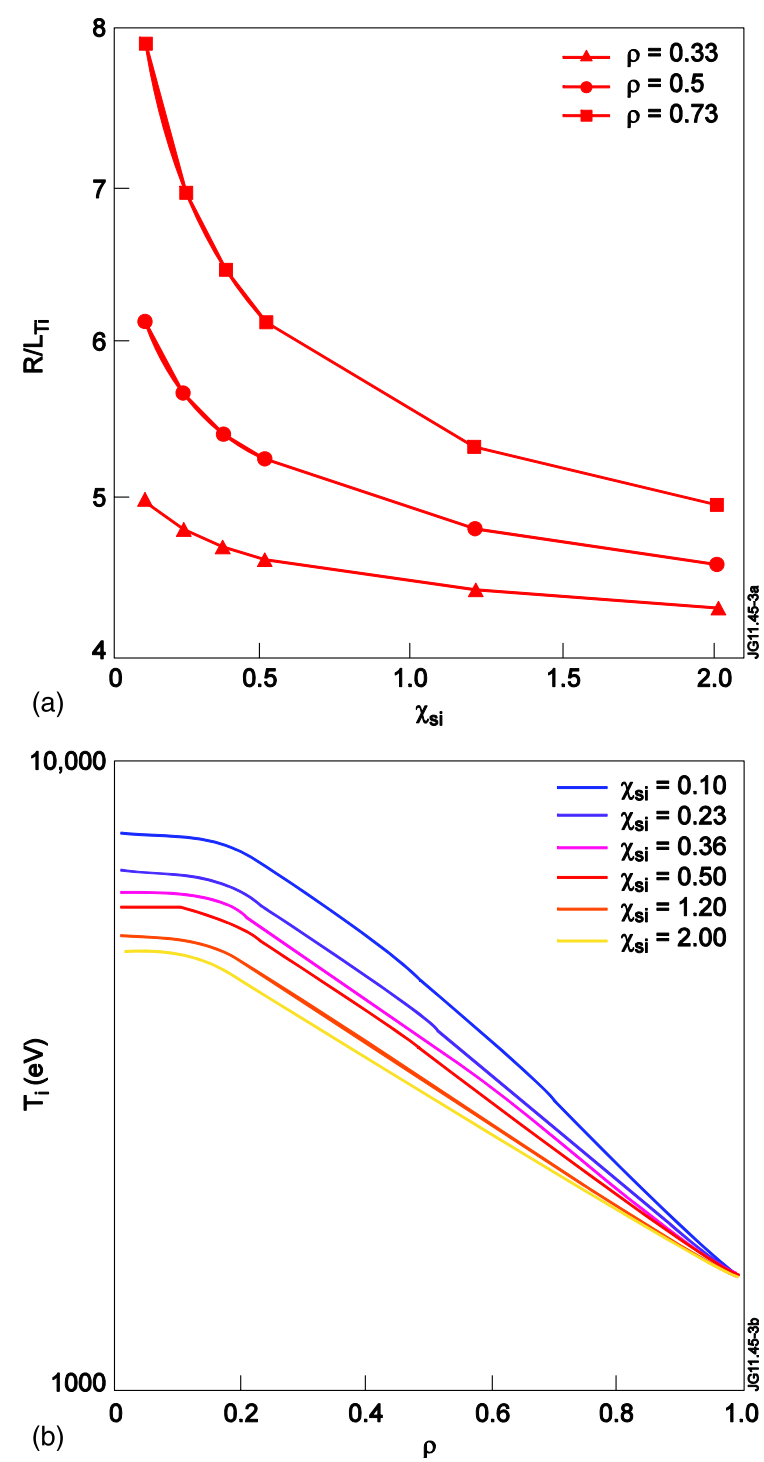

Figure 3. (a) $R / L_{T_{\mathrm{i}}}$ as a function of the ion stiffness level $\chi_{\mathrm{si}}$ is shown for different values of the radial coordinate. It refers to the case of typical hybrid plasma power and temperature pedestal height, and ion threshold $=4$, represented by the solid line with squares of the graph of figure 2. Triangular indicators are for $\rho=0.33$, circles for $\rho=0.5$, squares for $\rho=0.73$, where $\rho$ is the normalized toroidal minor radial coordinate. (b) Corresponding ion temperature profiles on logarithmic scale; different colours are used for the different values that ion stiffness reaches in the scan.

The effect of the threshold variation on $R / L_{T_{\mathrm{i}}}$ and on the ion temperature profiles are shown in figure 5 in the case of low constant stiffness, typical hybrid plasma temperature pedestal height $(1.5 \mathrm{keV})$ and power of $25.5 \mathrm{MW}$. In figure $5(a)$ we can see that $R / L_{T_{\mathrm{i}}}$ is not always higher than the chosen threshold of the scan, represented by the $x$-axis. In particular, it occurs for high values of ion threshold. In figure 5(b) we can see that for a higher ion threshold the ion temperature profiles reach higher values. However, the ion threshold growth leads to increasingly larger central region below threshold that prevents core temperatures to increase further. In fact, the injected power is not enough to give $R / L_{T_{\mathrm{i}}}$ values of the core region above the threshold, and therefore, a wider central zone of the plasma is dominated by neoclassical transport. Looking 


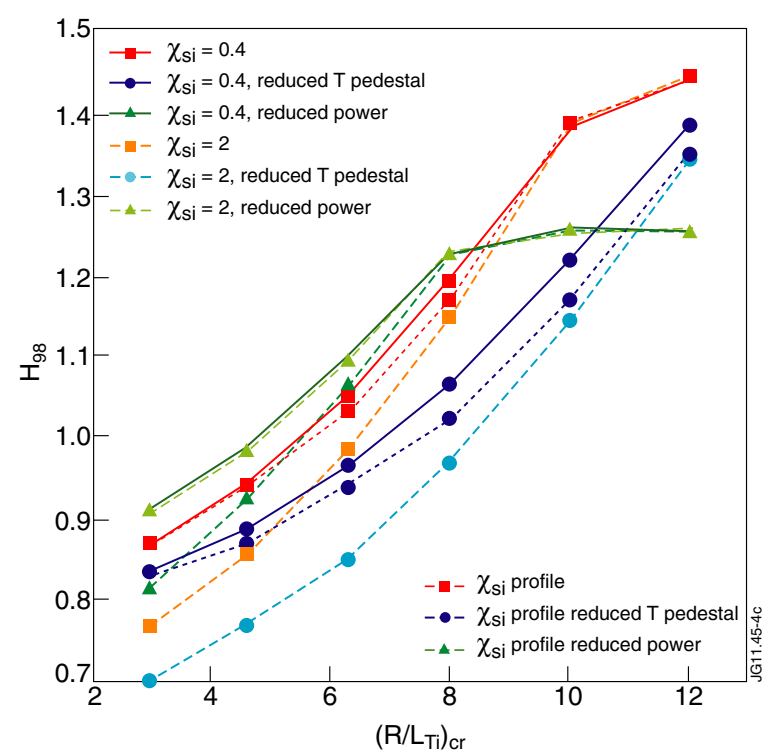

Figure 4. $H$ factor as a function of the ion threshold $\left(R / L_{T_{\mathrm{i}}}\right)_{\mathrm{cr}}$ for low constant ion stiffness (solid lines), for high constant ion stiffness (dashed lines), for the stiffness profile represented in figure 1 (dotted lines). With circles the case for typical hybrid plasma temperature pedestal and power is shown, with squares the case for reduced temperature pedestal, and with triangles the case for reduced power.

at figure 4 the increase in $H_{98}$ is lower over a certain value of the ion threshold for the case of a typical hybrid pedestal height and power (the same as for figure 5) and especially for the case of reduced power, for which similar behaviour takes place starting from lower values of threshold. It does not happen reducing the height of the temperature pedestal because lower ion temperature values are obtained, and, for ion thresholds up to 12 , the injected power seems to be sufficient to drive turbulent ion transport. For ion thresholds less than 7 we can note a larger increase in $H_{98}$ for all cases. Looking at figure $5(a)$, where $R / L_{T_{\mathrm{i}}}$ is shown as a function of the ion threshold for different radii in the case of typical hybrid temperature pedestal height $(1.5 \mathrm{keV})$ and power $(25.5 \mathrm{MW})$, it is evident that $R / L_{T_{\mathrm{i}}}$ is always higher than the corresponding abscissa for ion threshold lower than 7; its growing is higher for the inner radius. In figure $5(b)$ the corresponding ion temperatures are represented. In the plasma centre the ion temperature varies from $5.4 \mathrm{keV}$ for $\left(R / L_{T_{\mathrm{i}}}\right)_{\mathrm{cr}}=3$ to $9 \mathrm{keV}$ for $\left(R / L_{T_{\mathrm{i}}}\right)_{\mathrm{cr}}=7$.

Then the scan of temperature pedestal height was performed where we have changed both ion and electron temperature pedestal values. Different values of the power, the ion stiffness and the ion threshold have been considered. In the graph of figure 6 we can see that the $H$ factor increases with increasing temperature pedestal height: as expected [3133 ] it plays a very important role in increasing $H_{98}$. We can note that for reduced power (lines with triangles) the increase in the $H$ factor is slightly higher. Choosing different values for the fixed threshold and stiffness does not lead to large changes either: the case with stiffness and threshold profiles (dotted lines) gives slightly larger variation in the $H$ factor, which also assumes little higher values. We can state that $H_{98}$ varies about $75-80 \%$ on changing the temperature pedestal from 0.1 to $3 \mathrm{keV}$.
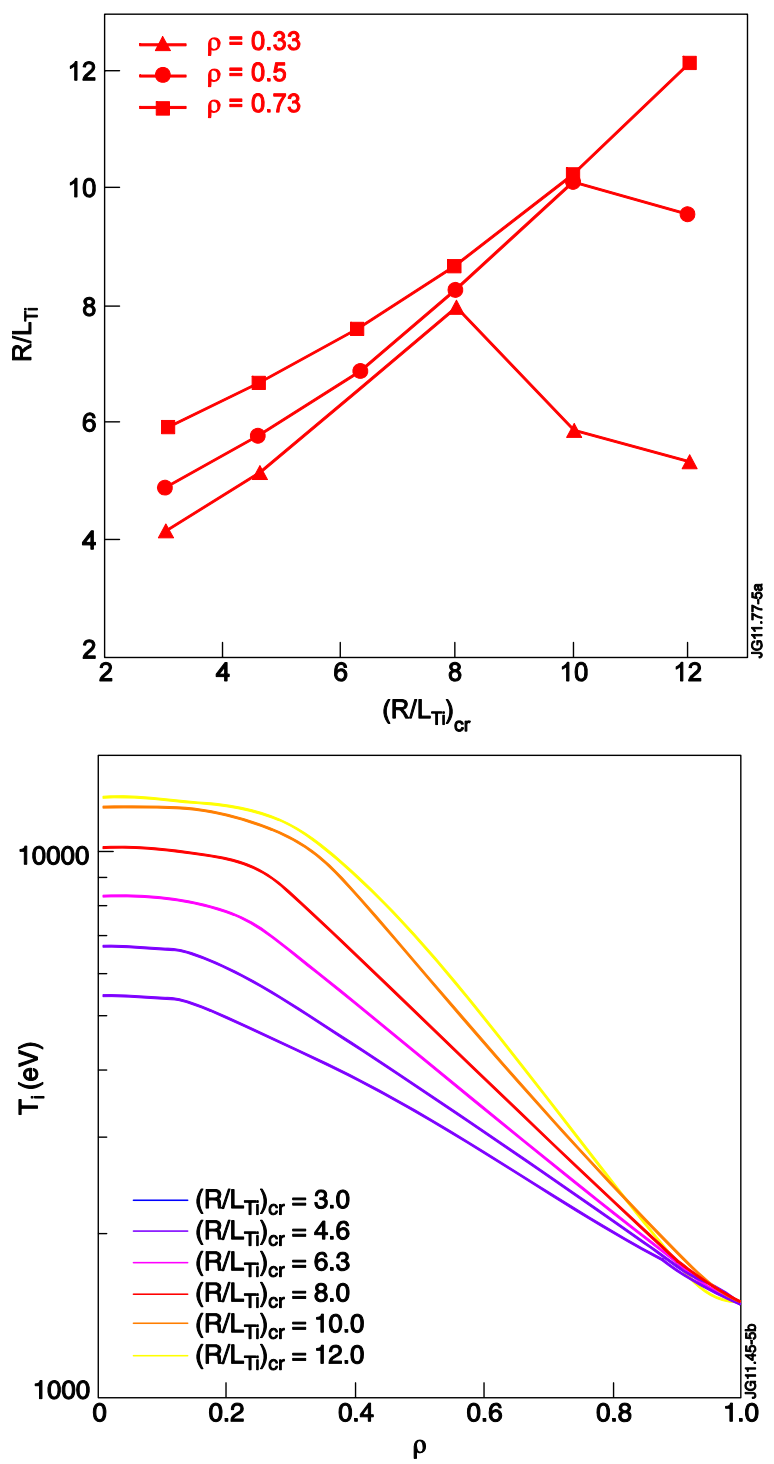

Figure 5. (a) $R / L_{T_{\mathrm{i}}}$ as a function of the ion threshold $\left(R / L_{T_{\mathrm{i}}}\right)_{\mathrm{cr}}$ is shown for different values of the radial coordinate. It refers to the case of ion stiffness $=0.4$, typical hybrid plasma temperature pedestal height and power, represented by the solid line with squares in the graph of figure 4 . Different curves in the graph refer to different radial positions. Triangular indicators are for $\rho=0.33$, circles for $\rho=0.5$, squares for $\rho=0.73$. (b) Correspondent ion temperature profiles are shown in logarithmic scale: different colours are used for the different values that ion threshold reaches in the scan.

The behaviour of the ion temperature profiles and their relative gradients with varying temperature pedestal height can be seen in figure 7. The graphs refer to the case of low constant stiffness (equal to 0.4 ), constant threshold (equal to 4 ) and typical hybrid plasma power of 25.5 MW. In figure 7(a) we can see that $R / L_{T_{\mathrm{i}}}$ is above the threshold for each radius whilst varying the temperature pedestal height. The corresponding variation of the $T_{\mathrm{i}}$ profiles is shown in figure $7(b)$ in which the central temperature changes from $4 \mathrm{keV}$ for a pedestal of $100 \mathrm{eV}$ to $9.8 \mathrm{keV}$ for a pedestal of $3 \mathrm{keV}$.

Finally, we did the power scan. The results are shown in figure 8 .

We can see that in the case of low stiffness (figure 8(a)) the $H$ factor does not depend on the power value. It is 


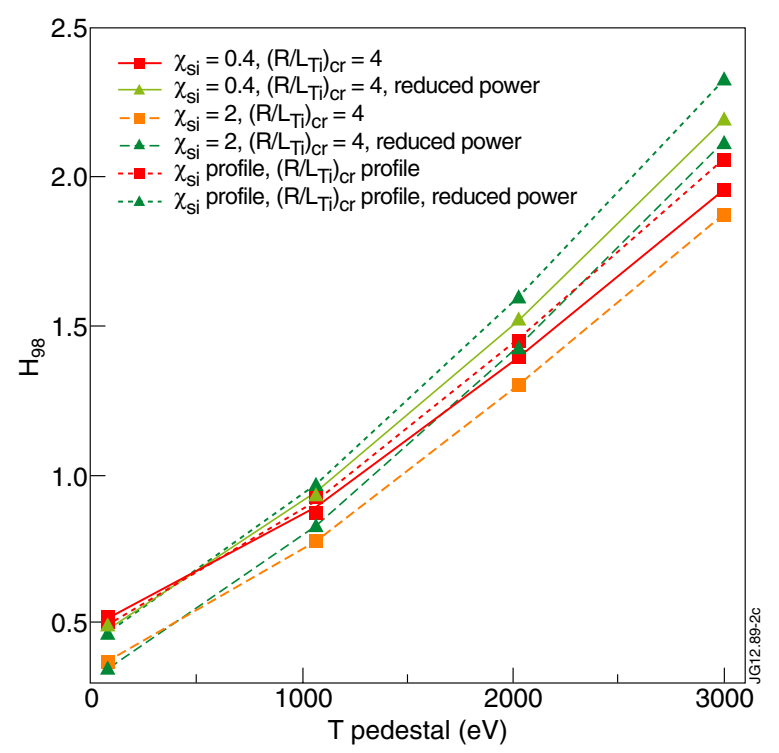

Figure 6. $H$ factor as a function of the ion and electron temperature pedestal height, for low constant ion stiffness and constant ion threshold (solid lines), for high constant ion stiffness and constant ion threshold (dashed lines), for the stiffness and threshold profiles represented in figure 1 (dotted lines). With squares we have represented the case of typical hybrid plasma power, with triangles the case of reduced power.

true for different fixed thresholds and temperature pedestal heights. Then the dependence on the injected power of energy confinement time for the hybrid JET plasmas characterized by low stiffness seems to be well reproduced by the energy confinement time obtained from the experimental scaling for $\mathrm{H}$-mode plasmas. In addition, we note that lowering the pedestal height from the typical hybrid plasma value of 1.5 to $1 \mathrm{keV}$ reduces the confinement less than lowering the threshold from 7 to 4 . In the case of high stiffness $H_{98}$ decreases with increasing power for a given set of temperature pedestal and threshold, as shown in figure $8(b)$. Roughly $10 \%$ loss of $H_{98}$ (coming from core confinement) is predicted on typical JET power levels when going from 20 to $30 \mathrm{MW}$ at high stiffness. The values of the $H$ factor and its drop with increasing power are larger for higher threshold and typical hybrid plasma temperature pedestal height.

The behaviour of the $T_{\mathrm{i}}$ profiles variation with changing power is represented in figure 9. It is shown for the case for high constant threshold, typical hybrid plasma temperature pedestal height. We can see that in figure $9(a)$, where the stiffness is low, the ion temperature assume values from 9.2 to $17.1 \mathrm{keV}$ in the centre of the plasma when varying the power. Instead in figure $9(b)$, for high stiffness, the values vary from 8 to $10.6 \mathrm{keV}$, and only in the very centre, where transport is below the threshold.

\section{Stiffness, ion threshold and pedestal scans in ITER plasmas}

\subsection{Set-up simulation}

In order to apply the scan analysis to ITER plasmas a standard $\mathrm{H}$-mode ITER simulation [34] has been used as the basis of
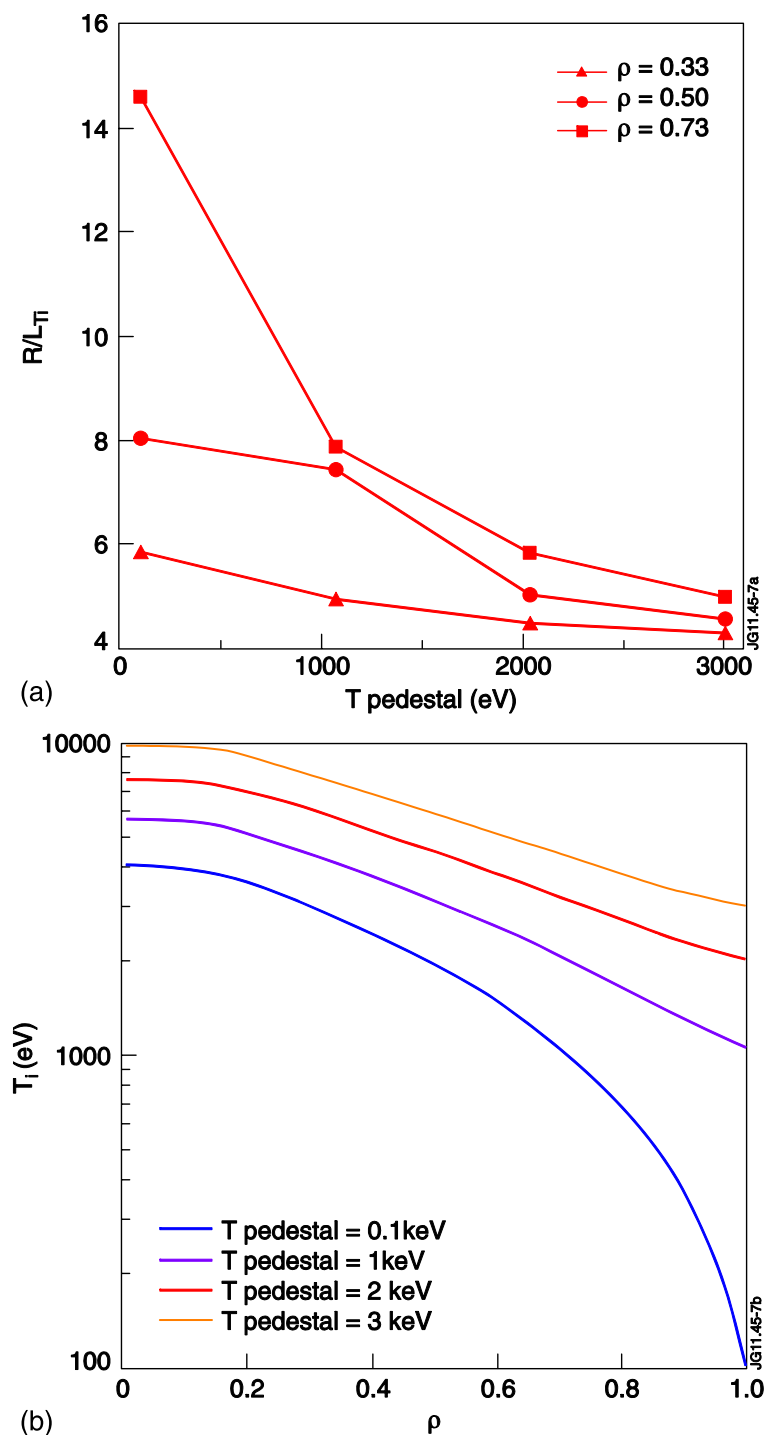

Figure 7. (a) $R / L_{T_{\mathrm{i}}}$ as a function of the ion and electron temperature pedestal height is shown for different values of the radial coordinate. It refers to the case of constant ion threshold (equal to 4), hybrid plasma power and stiffness $=0.4$, represented by the solid line with squares in the graph of figure 6 . Different curves in the graph refer to different radial positions. Triangular indicators are for $\rho=0.33$, circles for $\rho=0.5$, squares for $\rho=0.73$. (b) Corresponding ion temperature profiles are shown in logarithmic scale: different colours are used for the different values that the $T$ pedestal reaches in the scan.

the numerical simulations. The baseline ITER parameters have been taken: $B_{\mathrm{T}}=5.3 \mathrm{~T}, I_{\mathrm{P}}=15 \mathrm{MA}, R=6.2 \mathrm{~m}$, $a=2 \mathrm{~m}, n_{\mathrm{e} 0}=1.05 \times 10^{20} \mathrm{~m}^{-3}, q_{0}=0.75, Z_{\mathrm{eff}}=1.65$. We have scanned three parameters: the ion stiffness, the ion threshold and the ion and electron temperature pedestal height. The input power has been taken as fixed: $P_{\mathrm{NBI}}=33 \mathrm{MW}$, $P_{\text {ICRH }}=20 \mathrm{MW}$. NBI power deposition profiles have been calculated self-consistently by PENCIL [25]. For ICRH the RF power deposition profiles have been obtained by the code PION [35]. As in the case of hybrid JET plasmas only one parameter varies for each scan, the others are kept unchanged. When the temperature pedestal height is taken constant we have chosen the lower value of $3 \mathrm{keV}$ or the higher value of $4 \mathrm{keV}$. For fixed threshold and stiffness we proceeded as 

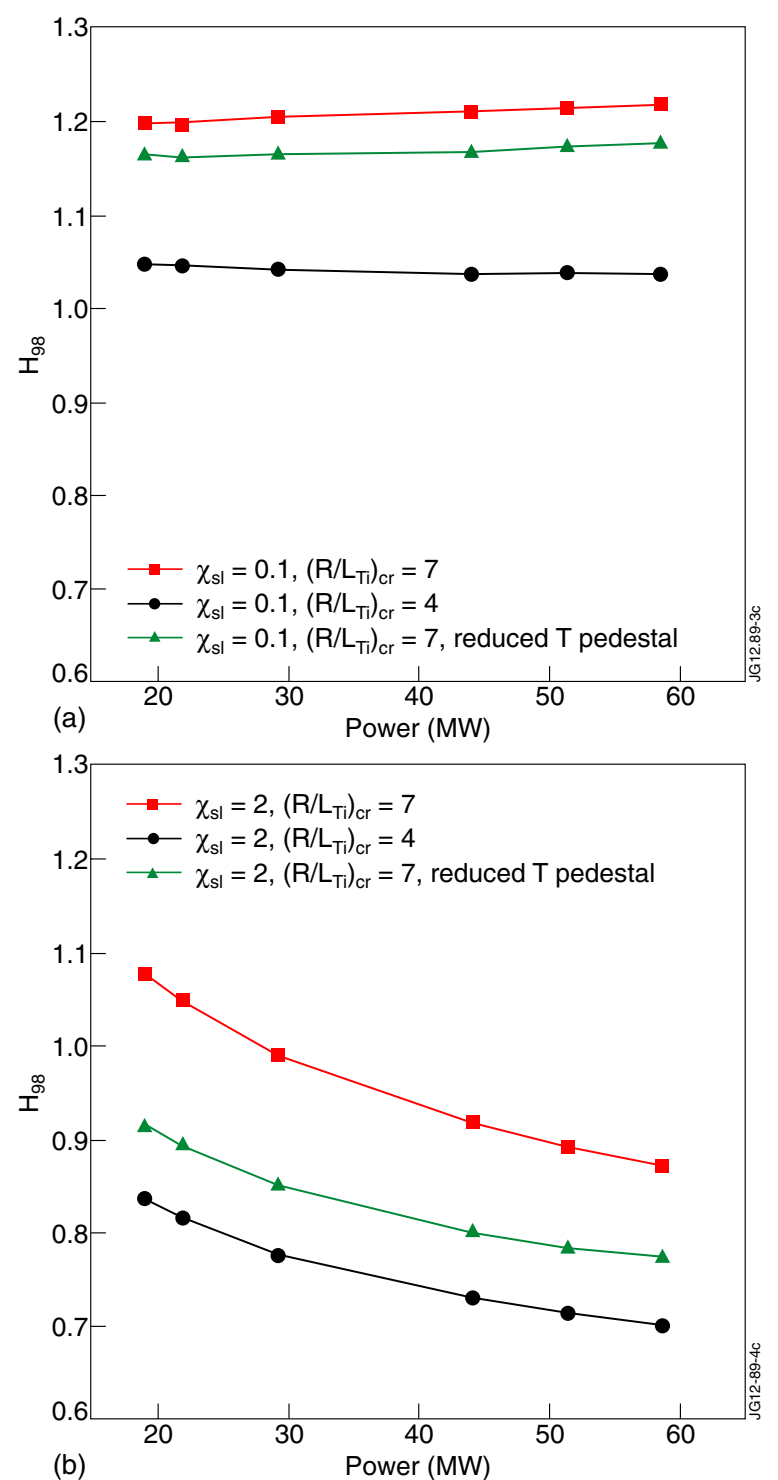

Figure 8. $H$ factor as a function of the power, $(a)$ for low constant stiffness (equal to 0.1 ), $(b)$ for high constant ion stiffness (equal to 2 ). With squares we have represented the simulations with typical plasma hybrid temperature pedestal height and high constant ion threshold (equal to 7), with circles the case of typical plasma hybrid temperature pedestal height and low constant threshold (equal to 4), and with triangles reduced pedestal height and high constant ion threshold (equal to 7).

for the simulations of the JET discharge (section 3.1), using radially constant values for electrons (electron stiffness $=1$, electron threshold $=5$ ) and both radially constant values (ion stiffness $=0.4$ or 2 , ion threshold $=4$ ) and radially dependent values (ion stiffness and threshold profiles of figure 1) for ions. A list of the made scans is shown in table 2.

\subsection{Simulation results}

For ITER plasmas the impact of the variation of each scanned quantity on global plasma performance is studied through the behaviour of the $H$ factor and also of the fusion power obtained from the simulations. First we proceeded with the ion temperature gradient stiffness scan, using different values

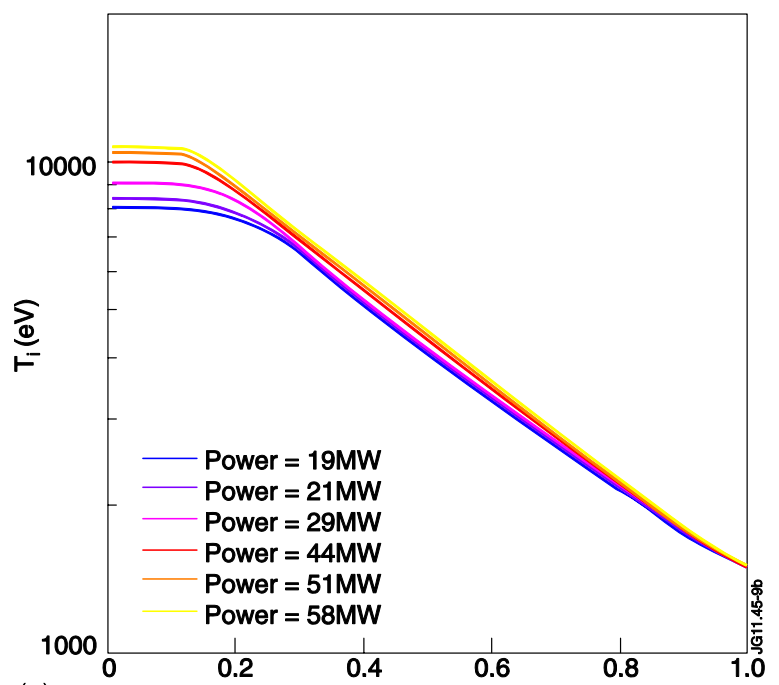

(a)

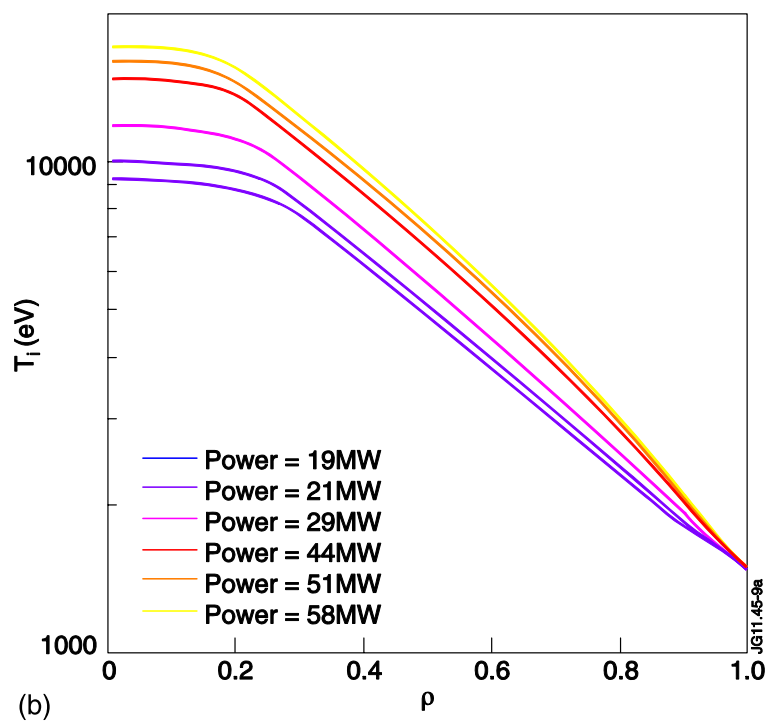

Figure 9. Ion temperature profiles in logarithmic scale are shown, (a) for low constant stiffness (equal to 0.1), (b) for high constant stiffness (equal to 2); both the graphs refer to the case with high constant threshold (equal to 7) and typical hybrid plasma pedestal height (equal to $1.5 \mathrm{keV}$ ). Different colours are used for the different values that power reaches in the scan.

of ion and electron temperature pedestal height and different ion thresholds. $H_{98}$, shown in figure $10(a)$ as a function of the ion stiffness, has the same behaviour as in the JET hybrid plasmas, but it is less sensitive to stiffness in ITER than in JET. In fact, in this case the difference caused by changes in the stiffness is less than $10 \%$. This is true with a constant fixed value of the ion threshold and the variation is even lower with the threshold profile. For the temperature pedestal height equal to $3 \mathrm{keV}$ (lines with circles) we find an $H$ factor more sensitive to stiffness changes, though with a lower value with respect to the case with higher pedestal. In figure $10(b)$, the fusion power as a function of the ion stiffness is shown.

We can see that changes in the stiffness cause an important variation of the fusion power, of over $50 \%$ of the total value, and it reaches up to $60 \%$ fixing the ion threshold constant and equal to 4 in the case of high pedestal height, and for low pedestal height with both threshold profile and constant threshold. 
Table 2. Scans of ion stiffness, ion threshold and temperature pedestal for ITER plasmas.

\begin{tabular}{|c|c|c|c|}
\hline & $\begin{array}{l}\text { Ion } \\
\text { stiffness }\end{array}$ & $\begin{array}{l}\text { Ion } \\
\text { threshold }\end{array}$ & $\begin{array}{l}\text { Temperature } \\
\text { pedestal }\end{array}$ \\
\hline \multirow[t]{4}{*}{ Scan of ion stiffness } & From 0.1 to 2 & 4 & $3 \mathrm{keV}$ \\
\hline & From 0.1 to 2 & 4 & $4 \mathrm{keV}$ \\
\hline & From 0.1 to 2 & Profile & $3 \mathrm{keV}$ \\
\hline & From 0.1 to 2 & Profile & $4 \mathrm{keV}$ \\
\hline \multirow[t]{6}{*}{ Scan of ion threshold } & 0.4 & From 3 to 12 & $3 \mathrm{keV}$ \\
\hline & 0.4 & From 3 to 12 & $4 \mathrm{keV}$ \\
\hline & 2 & From 3 to 12 & $3 \mathrm{keV}$ \\
\hline & 2 & From 3 to 12 & $4 \mathrm{keV}$ \\
\hline & Profile & From 3 to 12 & $3 \mathrm{keV}$ \\
\hline & Profile & From 3 to 12 & $4 \mathrm{keV}$ \\
\hline \multirow[t]{3}{*}{$\begin{array}{l}\text { Scan of temperature } \\
\text { pedestal }\end{array}$} & 0.4 & 4 & $\begin{array}{l}\text { From } 2 \\
\text { to } 6 \mathrm{keV}\end{array}$ \\
\hline & 2 & 4 & $\begin{array}{l}\text { From } 2 \\
\text { to } 6 \mathrm{keV}\end{array}$ \\
\hline & Profile & Profile & $\begin{array}{l}\text { From } 2 \\
\text { to } 6 \mathrm{keV}\end{array}$ \\
\hline
\end{tabular}

Finally, we can see that the higher the temperature pedestal, the higher the value of fusion power, the difference being over $100 \mathrm{MW}$. The drastic increase in the fusion power caused by the low stiffness can be explained by the large variation of the ion temperatures with the ion stiffness shown in figure 11(b) and their $R / L_{T_{\mathrm{i}}}$ values of figure $11(a)$. These graphs refer to the case of ion threshold equal to 4 and temperature pedestal height of $3 \mathrm{keV}$. The shown $T_{\mathrm{i}}$ and $R / L_{T_{\mathrm{i}}}$ development is similar to the other cases represented in figure 10 .

The large growth of the ion temperatures with decreasing ion stiffness, which leads from $T_{\mathrm{i} 0}=14 \mathrm{keV}$ for $\chi_{\mathrm{si}}=2$ to $T_{\mathrm{i} 0}=27 \mathrm{keV}$ for $\chi_{\mathrm{si}}=0.1$, is due to a significant increase in the gradient of $T_{\mathrm{i}}$ in the external part of the core as in the case of JET hybrid discharge, but also to its larger growth in the core and in the central region of the plasma. While in JET $R / L_{T_{\mathrm{i}}}$ is under the ion threshold values in the very central region, in the ITER case we find $R / L_{T_{\mathrm{i}}}$ quite over it. This can be explained using the on-axis ICRH system in ITER, which is characterized by a deposition power maximum in the centre of the plasma. It is scheduled in ITER together with the NBI system that is expected to give a more radially distributed deposition power. The ICRH gives an important contribution to the increase in the ion temperatures, and then to the magnitude of the fusion power.

The different sensitivity of the $H$ factor between the two machines is due to the inclusion of the $\alpha$ power in the total power (that comprehends also ohmic power and heating power), on which $H_{98}$ depends as shown in formula (2). In fact, as we can see also from the large variation of the fusion power represented in figure $10(b)$, the $\alpha$ power contributes significantly to the total value of the power. For lower ion stiffness the ion temperature achieves higher values, then the $H$ factor increases because it is directly proportional to the thermal energy. However, the $\alpha$ power is also larger and it causes a reduction in the $H_{98}$ with respect to the case of JET hybrid plasmas, in which the $\alpha$ power is always equal to zero. This behaviour takes place for all the scans performed for ITER, where nuclear fusion reactions have been enabled.
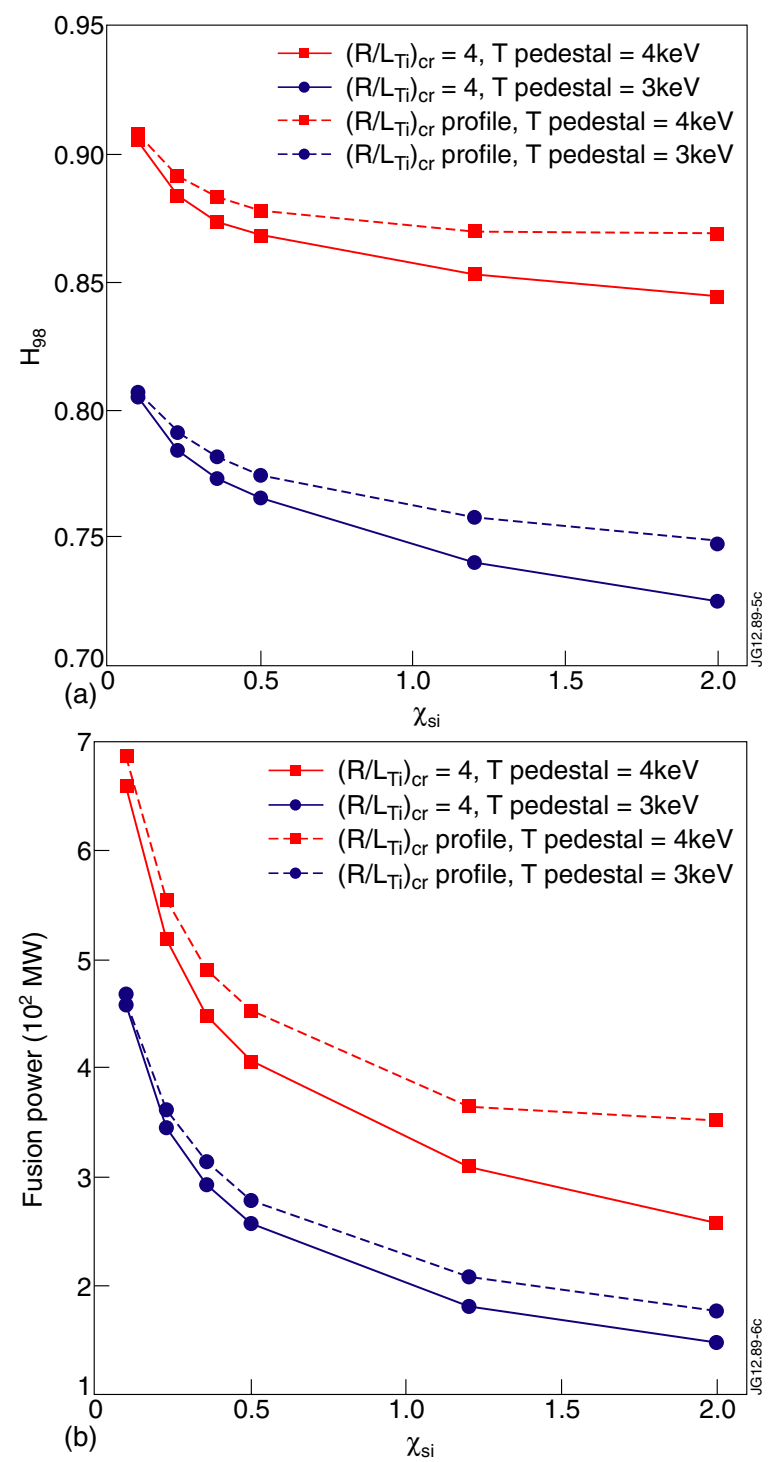

Figure 10. $H$ factor $(a)$ and fusion power $(b)$ shown as functions of the ion stiffness level $\chi_{\mathrm{si}}$. With circles we indicate the case with pedestal height equal to $3 \mathrm{keV}$, with squares the case with pedestal height equal to $4 \mathrm{keV}$. Solid lines are used for constant ion threshold (equal to 4), dashed lines for the ion threshold profile shown in figure 1 .

Then the ion temperature gradient threshold scan was made. In figure 12 the results are presented. In figure 12(a) we can see the $H$ factor as a function of the ion threshold for different values of ion stiffness and temperature pedestal. As for the scan of ion stiffness the variation of $\mathrm{H}_{98}$ is much lower with respect to the JET case, even if the behaviour is similar to the JET hybrid shot. Under the ion threshold value of 7 the $H$ factor increases with increasing threshold up to about $10 \%$, and its growth is larger $(11 \%)$ in the case of high ion stiffness (dashed lines). For values of the ion threshold higher than 7 the increase in $H_{98}$ is lower. As for JET hybrid simulations it corresponds to the $R / L_{T_{\mathrm{i}}}$ under threshold, that in the ITER case takes place in the outer region of the plasma, while in the centre it does not happen because of the ICRH power. The variation of the fusion power is larger for high constant ion stiffness too, as figure 12(b) shows: however, unlike the behaviour of the $H$ 

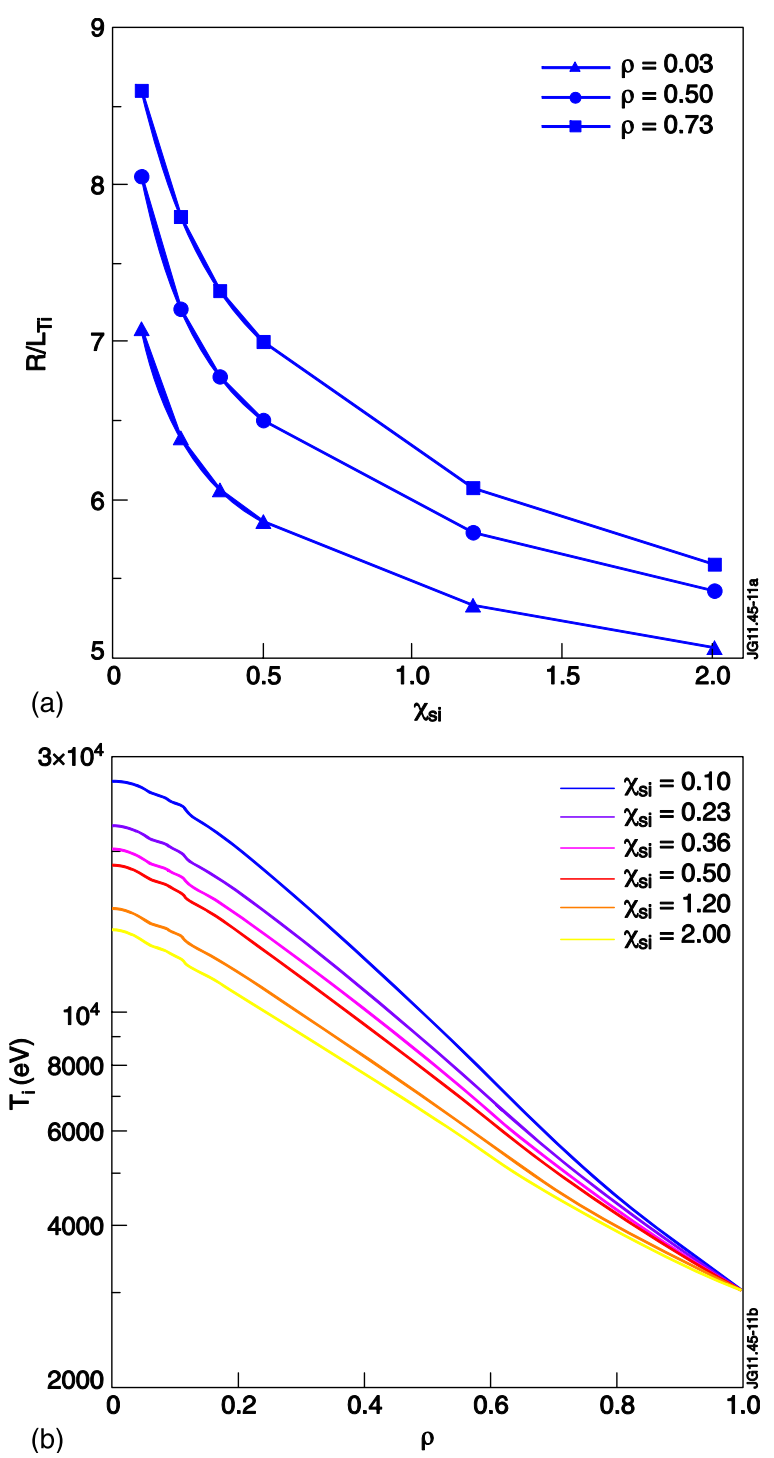

Figure 11. (a) $R / L_{T_{\mathrm{i}}}$ as a function of the ion stiffness is shown for different values of the radial coordinate. It refers to the case of constant ion threshold (equal to 4), power and temperature pedestal height $=3 \mathrm{keV}$, represented by the solid line with circles in the graphs of figure 10. Different curves in the graph refer to different radial positions. Triangular indicators are for $\rho=0.33$, circles for $\rho=0.5$, squares for $\rho=0.73$. (b) Corresponding ion temperature profiles are shown in logarithmic scale: different colours are used for the different values that the ion stiffness reaches in the scan.

factor, the impact of the ion threshold on the fusion power is considerable and it gives a variation of over $50 \%$. In addition, if the temperature pedestal height is equal to $4 \mathrm{keV}$ the value of the fusion power is higher by almost $100 \mathrm{MW}$.

Finally, we did the scan of the ion and electron temperature pedestal height. In figure $13(a)$ we can see the $H$ factor as a function of the temperature pedestal height. Also in this scan the increase in $H_{98}$ has the same behaviour as the case of the JET shot. The results shown in figure 13 agree with the previous scans about the role of the temperature pedestal height on ITER fusion power and confinement [36,37]. $H_{98}$ grows more than $30 \%$ with increasing temperature pedestal from 2 to $6 \mathrm{keV}$, achieving a variation of $40 \%$ for high stiffness. In figure $13(b)$ the huge growth of the fusion power with
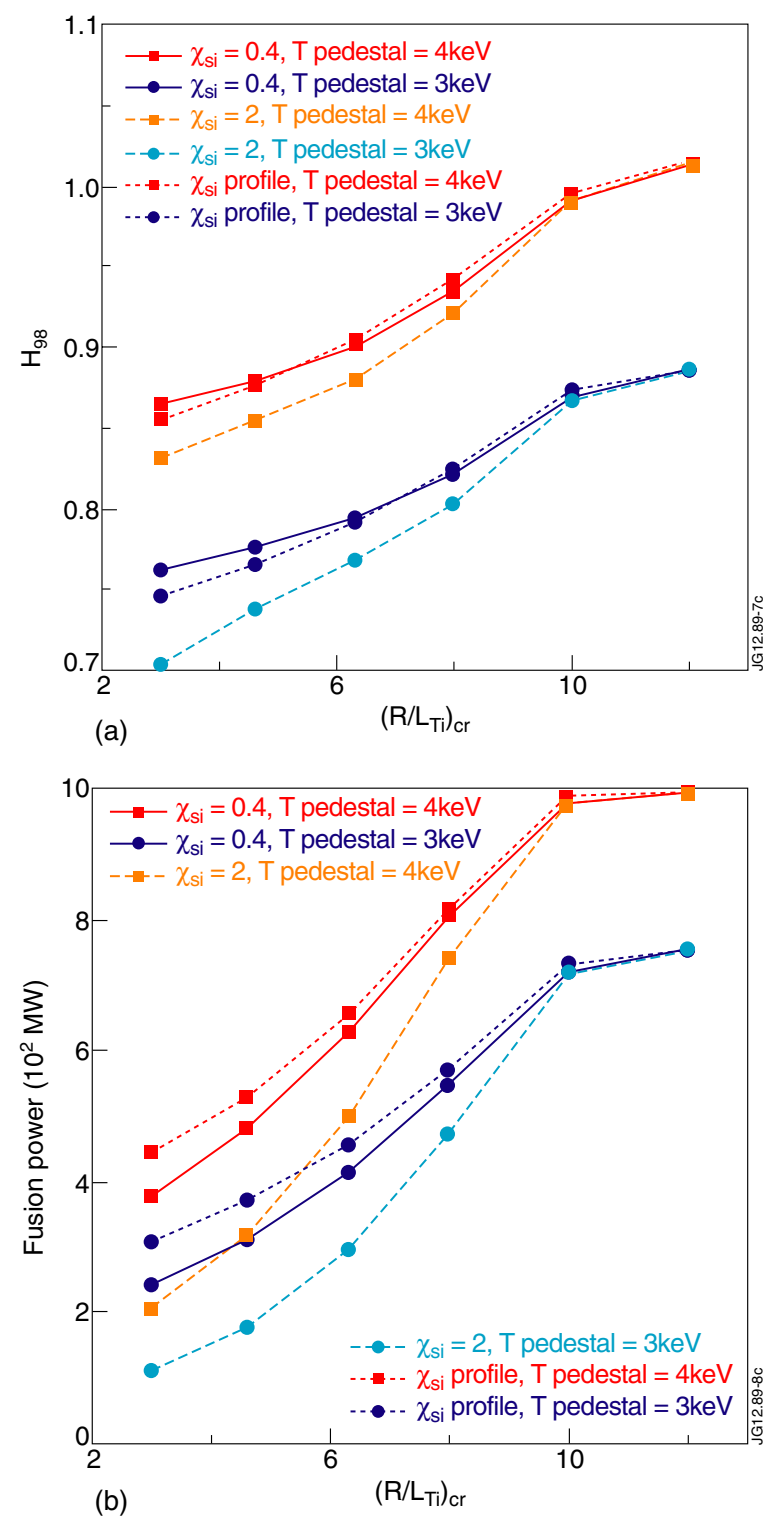

Figure 12. $H$ factor $(a)$ and fusion power $(b)$ shown as functions of the ion threshold. With squares (circles) colours we indicate the case with pedestal equal to $4 \mathrm{keV}(3 \mathrm{keV})$. Solid lines are for low constant ion stiffness, dashed lines for high constant ion stiffness, dotted lines for the ion stiffness profile represented in figure 1.

increasing pedestal height is shown. Over $80 \%$ of increase and values of fusion power almost of $1000 \mathrm{MW}$ are obtained for stiffness and threshold dependent on radial coordinate.

\section{Summary and conclusions}

A numerical simulation work has been carried out in order to investigate the role of the parameters connected with the turbulent transport driven by local micro-instabilities on the global plasma performance for hybrid JET plasmas and for ITER plasmas. Series of scans of ion stiffness, ion temperature gradient threshold, temperature pedestal height and injected power have been performed to study their impact on plasma performance and confinement. The fluid transport code JETTO has been used together with the semi-empirical 


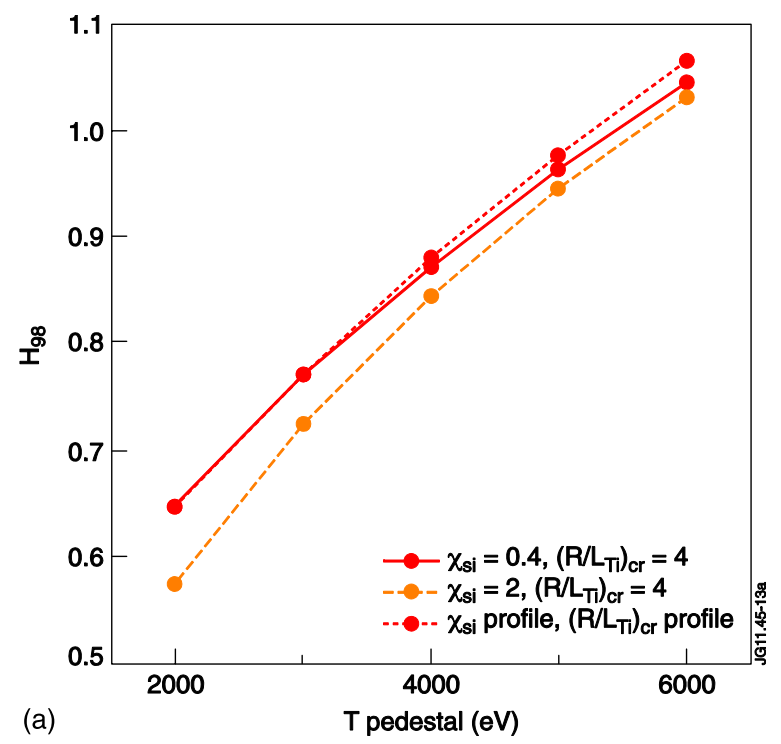

(a)

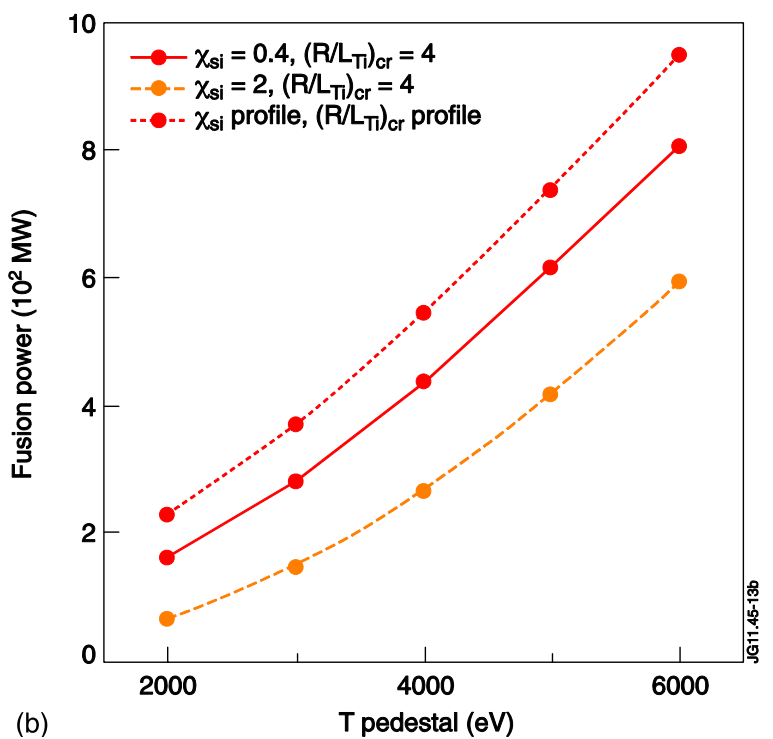

Figure 13. The $H$ factor $(a)$ and the fusion power $(b)$ are shown as functions of the ion and electron temperature pedestal height. With solid lines we indicate the case with low constant ion stiffness (equal to 0.4 ) and constant ion threshold (equal to 4), with dashed lines we have used high constant ion stiffness (equal to 2 ) and constant ion threshold (equal to 4). With dotted lines the case of ion stiffness and ion threshold profiles (represented in figure 1) is shown.

transport model CGM, which includes explicitly the scanned control parameters of turbulent transport. It has been then possible to obtain a quantitative estimate of the impact of the local transport parameters on the core plasma transport and globally on the plasma.

The results in this paper show that changes up to $30 \%$ in the $H$ factor and thereby in the plasma confinement can be achieved by changing the ion stiffness from very low values $\left(\chi_{\mathrm{s}}=0.1\right)$ to high values $\left(\chi_{\mathrm{s}}=2\right)$. Variations between $20 \%$ and $30 \%$ have been obtained from the scan of the ion threshold. Changes of over $50 \%$ in the $H_{98}$ have been found by changing the temperature pedestal height. This last result is in agreement with previous experimental studies. The relevance of the ion stiffness variation can partly explain the core confinement improvement characteristic of JET hybrid plasmas in terms of a broader central region of low stiffness with respect to the standard $\mathrm{H}$-mode plasmas. From these results it appears that stiffness can be a useful knob on which to act through modifications of plasma parameters in order to improve scenario performance. Differently from pedestal or threshold, stiffness was not paid much attention so far in the quest for confinement optimization, and this paper shows that it is worth pursuing further the investigation of how to control it. Its reduction in fact would allow us to gain significantly on core confinement, alleviating the plasma-wall interaction problems connected with high pedestal values.

In addition, we have seen that in the case of low stiffness, for a given set of threshold and pedestal height, energy confinement time in the JET hybrid scenario plasmas seems to depend on the injected power exactly as the energy confinement time obtained from the IPB98 H-mode scaling law [27]. This does not happen if the ion stiffness is high. In this case the $H$ factor decreases with increasing input power. The power dependence of the $H_{98}$ scaling then seems not to describe high stiffness plasmas adequately.

The scans have then been extended to ITER plasmas. The $H$ factor has been found to be less sensitive to the ion stiffness and ion threshold variations than for JET hybrid plasmas. However, looking at the fusion power changes, the effect of the variation of these parameters is very relevant. The scan of the ion and electron pedestal height gives large changes in the $H$ factor also for ITER plasmas, and a huge variation of the fusion power. The lesser sensitivity of $H_{98}$ to the ion stiffness and threshold variation is due to the dependence of the $H$ factor on $W_{\text {tot }} / P_{\text {tot }}^{0.31}$, where $W_{\text {tot }}$ is the thermal energy content and $P_{\text {tot }}$ the total power. In fact, the inclusion of $P_{\alpha}$ in $P_{\text {tot }}$ leads to a partial compensation of the changes in $W_{\text {tot }}$, because $P_{\alpha}$ increases with growing $W_{\text {tot }}$. It also leads the ion stiffness variation in relation to temperature pedestal changes to have more impact on the confinement for the case of JET hybrid discharge simulations than for ITER simulations. However, the ratio between the energy content difference obtained changing the ion stiffness and the one calculated varying the temperature pedestal give greater results for the ITER case. Then this behaviour, together with the decrease in the $H$ factor with increasing injected power at high stiffness and described for hybrid plasmas, could lead to an inherently wrong $H_{98}$ scaling for ITER if it is characterized by a high stiffness plasma.

This numerical work has then pointed out that the dependence of the global plasma performance on the ion stiffness, the ion threshold and the temperature pedestal height is quantitatively significant, both for JET and ITER scenarios, considering the variation of the three scanned parameters within a realistic interval of values defined by previous experimental studies. In particular, these results have pointed out the potential gain in scenario performance coming from controlling the stiffness level, thus giving further motivation for new experimental efforts in order to investigate how to lower the ion stiffness level. In addition, we conclude that when predicting the performance of future machines such as ITER, it is very important to know the ion stiffness level of their plasma, beyond the ion threshold and the temperature pedestal 
values, while in present model validation efforts stiffness is rarely taken into account.

\section{Acknowledgments}

This work, supported by the European Communities under the contract of Association EURATOM/ENEA-CNR, was carried out within the framework of EFDA. The views and opinions expressed herein do not necessarily reflect those of the European Commission.

(C) Euratom 2012

\section{References}

[1] Horton W et al 1999 Rev. Mod. Phys. 71735

[2] Weiland J 2000 Collective Modes in Inhomogeneous Plasmas (Bristol: Institute of Physics Publishing)

[3] Garbet X et al 2004 Plasma Phys. Control. Fusion 46 B557

[4] Ryter F et al 2006 Plasma Phys. Control. Fusion 48 B453

[5] Mantica P and Ryter F 2006 C.R. Physique 7634

[6] Mikkelsen D R et al 2003 Nucl. Fusion 4330

[7] Tardini G et al 2002 Nucl. Fusion 42258

[8] Wolf R C et al 2003 Plasma Phys. Control. Fusion 451757

[9] Baker D R et al 2001 Phys. Plasmas 84128

[10] Baker D R et al 2003 Phys. Plasmas 104419

[11] Mantica P et al 2009 Phys. Rev. Lett. 102175002

[12] Mantica P et al 2011 Phys. Rev. Lett. 107135004

[13] Mantica P et al 2011 Plasma Phys. Control. Fusion 53124033

[14] Imbeaux F et al 2001 Plasma Phys. Control. Fusion 431503
[15] Cenacchi G and Taroni A 1988 JETTO: A freeboundary plasma transport code (basic version) Rapporto ENEA RT/TIB 1988(5)

[16] Garbet X et al 2004 Plasma Phys. Control. Fusion 461351

[17] Garbet X et al 1996 Phys. Plasmas 31898

[18] Lin Z et al 2002 Phys. Rev. Lett. 88195004

[19] Waltz R E et al 2006 Phys. Plasmas 13072304

[20] Dimits A M et al 2000 Phys. Plasmas 7969

[21] Mantica P et al 2008 Fusion Sci. Technol. 531152

[22] Gruber O et al 1999 Phys. Rev. Lett. 831787

[23] Gormezano C et al 2004 Plasma Phys. Control. Fusion $46 \mathrm{~B} 435$

[24] Mantica P et al 2010 Proc. 23rd IAEA Fusion Energy Conf. on Plasma Physics (Dajeon, Republic of Korea) EXC/9-2

[25] Challis C D et al 1989 Nucl. Fusion 29563

[26] Ryter F et al 2011 Nucl. Fusion 51113016

[27] ITER Physics Basis Document 1999 Nucl. Fusion 392175

[28] Joffrin E et al 2010 Proc.23rd IAEA Fusion Energy Conf. on Plasma Physics (Dajeon, Republic of Korea) EX/1-1

[29] Strintzi D, private communication

[30] Waltz R E et al 1994 Phys. Plasmas 12229

[31] Maggi C F et al 2007 Nucl. Fusion 47535

[32] Frassinett L et al 2010 Proc. 37th EPS Conf. on Plasma Physics (Dublin, Ireland, 21-25 June 2010) P1.1031

[33] Fishpool G M et al 1998 Nucl. Fusion 381373

[34] Shimada M et al 2007 Progress in the ITER Physics Basis Chapter: 1. Overview and summary Nucl. Fusion $44 \mathrm{~S} 1$

[35] Eriksson L G, Hellsten T and Willen U 1993 Nucl. Fusion 331037

[36] Kinsey J E et al 2003 Nucl. Fusion 431845

[37] Doyle E J et al 2007 Progress in the ITER Physics Basis: Chapter 2. Plasma confinement and transport Nucl. Fusion $47 \mathrm{~S} 18$ 